\title{
Automatisiertes Fahren als Herausforderung für Städte und Regionen
}

Eine interdisziplinäre Analyse denkbarer Anwendungen und Effekte

Fabienne Perret, EBP Schweiz AG, Geschäftsbereich Verkehr, Mühlebachstrasse 11, 8032 Zürich (fabienne.perret@ebp.ch)

Remo Fischer, EBP Schweiz AG, Geschäftsbereich Verkehr, Zürich (remo.fischer@ebp.ch)

Holger Frantz, EBP Deutschland GmbH, Berlin (holger.frantz@ebp.de)

Das automatisierte Fahren hat das Potenzial, unsere alltägliche Mobilität grundlegend zu verändern. Auf Basis eines denkbaren Entwicklungspfades werden die heute absehbaren Auswirkungen, insbesondere aus Sicht der Städte und Regionen, hergeleitet. Dabei stehen Chancen wie Effizienzgewinne, neue Mobilitätsangebote und eine Erhöhung der Verkehrssicherheit den Herausforderungen wie potenzielle Verkehrszunahmen, Anpassungen der Regulierungen oder der Aufbau einer Dateninfrastruktur gegenüber. Ob das automatisierte Fahren gesamthaft einen Nutzen bringen wird, hängt deutlich von der Einflussnahme der Politik ab.

\section{Automated driving as a challenge to cities and regions} An interdisciplinary analysis of potential applications and effects

Automated driving has the potential to fundamentally change our everyday mobility. Based on a conceivable development path, the effects that are foreseeable from today's perspective of cities and regions are described. Automated vehicles offer numerous opportunities such as efficiency gains, new mobility options, and increased safety. Upcoming challenges are potential traffic growth, necessary adjustments in legislation, and the development of an adequate data infrastructure. The overall benefit of automated driving strongly depends on political influence.

Keywords: automated vehicles, driverless cars, sharing services, public transportation, traffic management

This is an article distributed under the terms of the Creative Commons Attribution License CCBY 4.0 (https://creativecommons.org/licenses/by/4.0/)

https://doi.org/10.14512/tatup.27.2.31

Submitted: 09. 03.2018. Peer reviewed. Accepted: 11. 05.2018

\section{Hintergrund}

Das Thema automatisiertes Fahren wurde bisher vor allem durch Technologieunternehmen und Industrieunternehmen besetzt, die es primär mit Vernetzung, Sharing und Elektromobilität in Verbindung gebracht haben - nicht zuletzt, um sich aus Gründen des Marketings als Innovationsträger zu präsentieren. Gesichertes Wissen über die Wirkungen des automatisierten Fahrens auf das Mobilitätsverhalten, auf das gesamte Verkehrsgeschehen, auf die Raumentwicklung oder den Infrastrukturbedarf fehlt jedoch weitgehend. Diese Aspekte finden aktuell vermehrt Eingang in Forschungsbestrebungen.

Neben den Hochleistungsstraßen, die in besonderem Maße im Fokus der Bundesregierungen liegen (Deutsche Bundesregierung 2015; Schweizerischer Bundesrat 2016), stellen sich für die Planerinnen und Planer in den Städten und Regionen zentrale Fragen zum untergeordneten Verkehrsnetz: Welchen Einfluss haben automatisierte Fahrzeuge auf die Kapazität der Verkehrsnetze und das Erscheinungsbild der Mobilität im Siedlungsgebiet? Bedarf es neuer Infrastrukturen? Welches sind die Rollen und Verantwortlichkeiten der Kommunen und Städte? Wem gehören die produzierten Daten? Welche Mobilitätsangebote haben Marktchancen?

\section{Vorgehen}

Im Rahmen einer interdisziplinären Studie (EBP 2017), welche in Zusammenarbeit mit zahlreichen schweizerischen Akteuren (u. a. Städte, Kantone, Transportunternehmen) entstand, wurden die relevanten Aspekte des automatisierten Fahrens diskutiert und mögliche Auswirkungen abgeschätzt. Der gemeinsam festgelegte Fokus lag auf planerischen Aspekten des Personenverkehrs auf Schiene und Straße, rechtliche Fragen standen nicht im Zentrum. 
Das methodische Vorgehen umfasste zuerst eine Literaturanalyse zu den Definitionen und Wirkungsmechanismen. In interdisziplinären Workshops wurden mit den beteiligten Akteuren der Studie ausgewählte Fragestellungen vertieft und insbesondere der Entwicklungspfad erarbeitet. Auf dieser Grundlage konnten weitere Literaturanalysen zu ausgewählten Fragestellungen, quantitative Abschätzungen sowie gezielte Experteninterviews zur Vertiefung durchgeführt werden.

\section{Denkbarer Entwicklungspfad}

Für die Untersuchung wurde angenommen, dass die menschliche Innovationskraft das automatisierte Fahren früher oder später erfolgreich ermöglichen wird. Die Gesellschaft wird das automatisierte Fahren in der Zukunft als alltäglich betrachten und breit nutzen. Doch wie kommen wir dahin? Um die Auswirkungen über die Zeit beschreiben zu können, wurde für die Schweiz ein Entwicklungspfad mit sechs Zuständen skizziert. Er wird aus heutiger Sicht sowohl von den involvierten Akteuren als auch von den Autorinnen und Autoren als plausibel und wahrscheinlich eingeschätzt. Es wird dementsprechend nicht der Anspruch erhoben, dass dieser die einzig mögliche Entwicklung darstellt. Welche Abfolge tatsachlich eintreten wird, ist von vielen Faktoren abhängig. Der skizzierte Entwicklungspfad ermöglicht es jedoch, die Komplexität für Wirkungsanalysen zu reduzieren und die Handlungsoptionen in Schritten aufzuzeigen.

Grundsätzlich kann der Entwicklungspfad auf Deutschland adaptiert werden, allerdings müssen die unterschiedliche Bedeutung der Automobilindustrie (zahlreiche Automobilhersteller in Deutschland) und des öffentlichen Verkehrs (tieferer Nachfrageanteil in Deutschland als in der Schweiz) berücksichtigt werden. Der Pfad zeigt einen plausiblen Verlauf der Entwicklung in unterschiedlichen Teilräumen auf, er beinhaltet jedoch keine konkreten Zeithorizonte. Tabelle 1 gibt einen Überblick über die erarbeiteten sechs Zustände des Entwicklungspfads der Automatisierung nach Anwendungsbereichen.

\section{Motorisierter Individualverkehr}

Abbildung 1 zeigt die sechs Zustände des Entwicklungspfads der Automatisierung im motorisierten Individualverkehr (MIV). Dabei werden die internationalen Automatisierungsgrade (levels of automation) verwendet (SAE 2014). Der MIV dürfte eine schrittweise generische Entwicklung vom konventionellen selbstgesteuerten Fahrzeug bis hin zur vollständigen Automatisierung durchlaufen. Die nötigen Bewilligungen bzw. Zulassungen werden technisch und räumlich ausgeweitet - von der Sonderbewilligung für Teststrecken an einzelne Entwickler über die temporäre Freigabe von Pilotstrecken für Nutzer mit entsprechend zugelassenem Fahrzeug, hin zur allgemeinen Freigabe im gesamten Straßennetz.

Automatisierte Fahrzeuge dürften sich zuerst auf den Hochleistungsstraßen (Zustände 2 und 3 gemäß Abb. 1) und dann im städtischen Raum (Zustände 3 und 4 gemäß Abb. 1) durchsetzen. Der Betrieb des Autobahnnetzes als geschlossenes System ist einfacher: Die Fahrtrichtungen sind baulich getrennt, Konflikte der Trajektorien kommen nur in Bezug auf Spurwechselvorgänge vor und es sind keine anderen Verkehrsmittel vorhanden. In Siedlungsräumen ist die Situation aufgrund des Mischverkehrs mit anderen Verkehrsmitteln und den zahlreichen Fahrkonflikten zwar deutlich komplexer als außerorts. Die gefahrenen Geschwindigkeiten sind jedoch niedriger und damit die Möglichkeiten der Erfassungstechnologien in Bezug auf die Erkennung von sich bewegenden Objekten deutlich besser. Darüber hinaus dürfte die Datennetzabdeckung in Siedlungsräumen über lange Zeit besser sein als in ländlichen Gebieten. Deshalb wird gemäß Abbildung 1 erst ab Zustand 4 mit einer Anwendung des automatisierten Fahrens auf Überlandstraßen gerechnet.

\begin{tabular}{|c|c|c|c|}
\hline Zustand & Motorisierter Individualverkehr (MIV) & Straßengebundener ÖPNV & Schienenverkehr \\
\hline 1 & Fahrassistenzsysteme L1/L2 auf Gesamtnetz & Teststrecken (L4) & Isolierte Anwendungen (GoA 3/4) \\
\hline 2 & Freigabe L3 auf Hochleistungsstraßen & $\begin{array}{l}\text { Automatisierte Kleinbusse innerorts im Regel- } \\
\text { betrieb (L4) }\end{array}$ & Ausgewählte Teststrecken (GoA 3/4) \\
\hline 3 & $\begin{array}{l}\text { Freigabe L4 auf Hochleistungsstraßen, } \\
\text { L3 auf Straßen im Siedlungsraum }\end{array}$ & $\begin{array}{l}\text { Automatisierte Kleinbusse innerorts im Regel- } \\
\text { betrieb (L5) }\end{array}$ & \multirow[t]{2}{*}{$\begin{array}{l}\text { Automatisierter Zugbetrieb in Teilnetzen } \\
\text { (GoA 3/4) }\end{array}$} \\
\hline 4 & $\begin{array}{l}\text { Freigabe L4 auf Straßen im Siedlungsraum, } \\
\text { L3 auf Überlandstraßen }\end{array}$ & $\begin{array}{l}\text { Automatisierte Kleinbusse inner-/außerorts } \\
\text { im Regelbetrieb (L5) }\end{array}$ & \\
\hline 5 & $\begin{array}{l}\text { L4 auf Überlandstraßen und schließlich } \\
\text { L5 auf dem Gesamtnetz }\end{array}$ & $\begin{array}{l}\text { Alle Fahrzeuge inner-/außerorts im Regel- } \\
\text { betrieb (L5), adaptiver/linienfreier ÖPNV } \\
\text { (insb. auf nachfrageschwachen Beziehungen) }\end{array}$ & \multirow[t]{2}{*}{$\begin{array}{l}\text { Automatisierter Zugbetrieb im Gesamtnetz } \\
(\text { GoA 3/4) }\end{array}$} \\
\hline 6 & $\begin{array}{l}\text { L5 auf dem Gesamtnetz, hoheitliche Einfluss- } \\
\text { nahme }\end{array}$ & Zusätzlich: Hoheitliche Einflussnahme & \\
\hline
\end{tabular}

Tab. 1: Kurzfassung der Entwicklungspfade über sechs Zustände hinweg für die drei genannten Anwendungsbereiche. Level (L) bzw. Grade of Automation (GoA) geben die Automatisierungsstufen gemäß SAE- bzw. UITP-Standards an. 


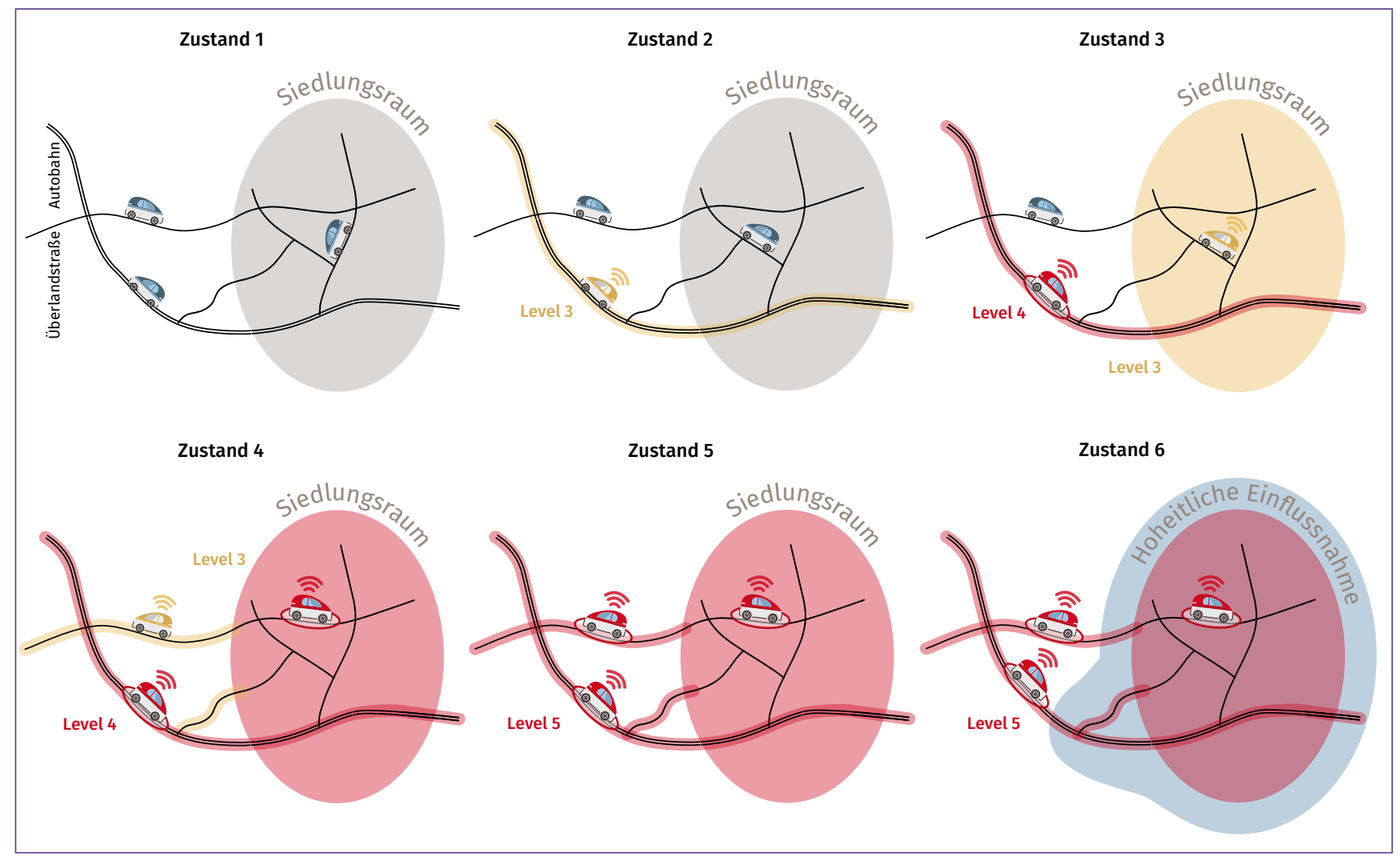

Abb.1: Entwicklungspfad beim MIV (Zustände 1-6).

Quelle: Eigene Darstellung

Mit zunehmender Automatisierung wird das Mobilsein attraktiver, unter anderem weil die Reisezeit im Auto für andere Aktivitäten genutzt werden kann. Dies dürfte die Verkehrsnachfrage deutlich steigern, wie die detaillierte Auswirkungsanalyse im Abschnitt „Auswirkungen“ aufzeigt. Da der Spielraum für einen Ausbau der Verkehrsinfrastrukturen in der Schweiz sehr begrenzt ist und eine ungebremste Zunahme des Verkehrsaufkommens politisch nicht erwünscht ist, drängen sich Lenkungsinstrumente auf. Auf Basis einer Iteration wurde daher im letzten Zustand des Entwicklungspfades (Zustand 6 gemäß Abb. 1) von einer staatlichen Einflussnahme ausgegangen, welche das Mobilitätsverhalten mit (neuen) Regulativen und Anreizsystemen in eine nachhaltige Richtung steuert. Davon können alle Verkehrsmittel betroffen sein. Zur Einflussnahme gehören beispielsweise Anreize zur Bündelung von Wegen oder die Beschränkung von Leerfahrten. Die Anwendung solcher Instrumente kann auch schon in früheren Zuständen erfolgen, wurde hier aber zur differenzierten Folgenabschätzung bewusst an das Ende des Entwicklungspfades gestellt.

\section{Straßengebundener ÖPNV}

Beim straßengebundenen öffentlichen Personennahverkehr (ÖPNV) bieten sich infolge der Automatisierung Möglichkeiten für veränderte Transportangebote (UITP 2017). Bereits heute werden in zahlreichen Städten automatisierte Minibusse mit Sonderbewilligungen und unter Begleitung getestet. Anwendungen dürften sich über den automatisierten Betrieb einzelner Linien, dem automatisierten Regelbetrieb im ÖPNV-Netz, hin zu einem adaptivem ÖPNV entwickeln. Dieser folgt keiner definierten Linienführung mehr, sondern führt bei jeder Fahrt ein optimiertes Routing zur Bündelung der angemeldeten Fahrtenwünsche der Kunden durch. Insbesondere in peripheren Stadtgebieten und im ländlichen Raum weist diese Angebotsform Vorteile auf (kostengünstige, gute Bedienung bei geringer Nachfrage). In dichten städtischen Räumen dürften auf Hauptachsen weiterhin große Fahrzeuggefäße im Linienverkehr zum Einsatz kommen, da sie in Bezug auf Kapazitäten und Bündelung kaum zu ersetzen sind.

\section{Car-Sharing und Pooling}

Im Übergangsbereich von MIV und ÖPNV besteht infolge des führerlosen Betriebs (mind. SAE-Level 4) das Potenzial für neue Verkehrsangebote. Dazu gehört einerseits das Car-Sharing, bei dem Fahrzeuge einer automatisierten Flotte individuell und seriell genutzt werden können. Andererseits werden auch Pooling-Angebote in der Form von automatisierten Sammeltaxis möglich, bei denen Fahrzeuge von verschiedenen Personen gleichzeitig genutzt werden und so ein Bündelungseffekt eintritt. Diese Sammeltaxis optimieren ihren Betrieb auf der Basis von angemeldeten Fahrten (on-demand). Sie können in den ÖPNV 


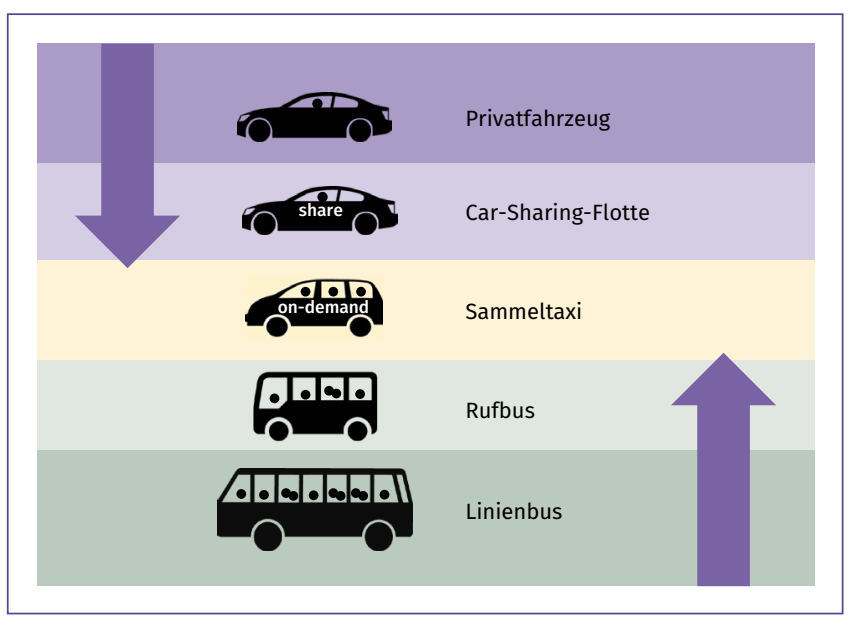

Abb.2: Angebotsformen zwischen MIV und ÖPNV.

Quelle: Eigene Darstellung

eingebunden sein oder von privaten Anbietern betrieben werden. Intensives Car-Sharing und Pooling bedingen einen Bedeutungsverlust des individuellen Fahrzeugbesitzes. Abbildung 2 gibt die beschriebenen Angebotsformen wieder, ohne auf die Abhängigkeiten von Gesamtflotten, Fahrzeuggröße, Fahrzeugbetrieb oder Routingprinzipien einzugehen. Diese sind Bestandteil von anderen Forschungsarbeiten (z. B. OECD/ITF 2015).

\section{Schienengebundener Öffentlicher Verkehr (ÖV)}

Auch für den schienengebundenen ÖV wurde im Rahmen der Studie ein Entwicklungspfad erarbeitet, der von bereits heute vorhandenen netztopologisch isolierten Anwendungen (z. B. führerlose U-Bahnen) hin zu einem automatisierten, führerlosen Zugbetrieb im gesamten Schienennetz führt. Die Abfolge der Zustände wird dabei als weniger komplex als im Straßenverkehr angenommen, da die Fahrzeuge fixe Strecken befahren, heute schon in übergeordnete Steuerungen eingebunden sind und als Flotten beschafft sowie erneuert werden. Wie rasch sich führerloses Fahren auf der Schiene durchsetzt, ist aber insbesondere auch von Akzeptanzfragen bei den Nutzenden und von den langlebigen Fahrzeuggenerationen abhängig. Das ÖV-Rollmaterial ist deutlich länger im Einsatz als im Individualverkehr.

\section{Auswirkungen}

\section{Faktoren für die Marktdurchdringung automatisierter Fahrzeuge}

Im Hinblick auf eine Abschätzung der verkehrlichen Wirkungen muss analysiert werden, wie sich automatisierte Fahrzeuge in der Gesamtflotte über die Zustände verbreiten werden. Dabei sind insbesondere die individuellen Zusatznutzen als auch die Zusatzkosten entscheidend. Zusatznutzen entstehen für die Käufer hinsichtlich der Sicherheit. In den ersten Zuständen geht das automatisierte Fahren vor allem mit einer höheren passiven Sicherheit einher, da Eingriffe in Längs- und Querführung des
Fahrzeuges das Ausmaß und die Folgen der Unfälle reduzieren. In einer zweiten Phase mit zunehmender Vernetzung zwischen den Fahrzeugen wird durch die weitgehende Vermeidung von Unfällen auch die aktive Sicherheit erhöht. Ein weiterer wichtiger Nutzen entsteht durch den Zeitgewinn. In den ersten $\mathrm{Zu}-$ ständen des automatisierten Fahrens kann ein Teil der Unterwegszeit für alternative Tätigkeiten genutzt werden. In den $\mathrm{Zu}-$ ständen mit Vollautomatisierung können Fahrzeuge führerlos unterwegs sein, was den Reisekomfort und damit den Nutzen weiter erhöht und eine zeitliche Flexibilität dank der Möglichkeit von Fahraufträgen bringt. Die Zusatzkosten sind vor allem durch die erhöhte technische Ausstattung der Fahrzeuge sowie die zunehmenden Kommunikationskosten (Datenübertragung in Echtzeit) begründet.

Bei der Abschätzung der Marktdurchdringung ist zu berücksichtigen, dass auch künftig ein Unterschied zwischen den technischen Möglichkeiten und den gesetzlich erlaubten Funktionen bestehen wird. Fahrzeuge werden voraussichtlich bis zur Erreichung von Zustand 4 mehr können als sie dürfen. Das heißt im Umkehrschluss: Nur, weil die technologischen Voraussetzungen gegeben sind, kann nicht auch mit einer raschen Verbreitung des automatisierten Fahrens gerechnet werden - weder im ÖV noch beim MIV. Der Unterschied ist im heutigen Zustand 1 noch bescheiden, dürfte jedoch in den Zuständen 2 und 3 recht erheblich sein (Abb. 3).

\section{Einfluss auf die Verkehrsnachfrage}

Automatisierte Fahrzeuge verändern das gesamte Verkehrssystem und damit die verkehrliche Nachfrage. Relevante Einflussfaktoren auf die Nachfrage wurden identifiziert und im Zusammenhang mit automatisiertem Fahren abgeschätzt.

Nutzung der Reisezeit: Durch die Automatisierung wird die Reisezeit anderweitig nutzbar. In den ersten Zuständen muss der Fahrer zur Übernahme der Fahraufgaben bereit sein (beispielsweise in Zustand 2 auf Hochleistungsstraßen), in den weiteren Zuständen ist diese Bereitschaft nicht mehr notwendig. Durch die Nutzung der Reisezeit sinken die individuellen Reisezeitkosten, weshalb allenfalls weiter entfernt gelegene Ziele angefahren werden. Reisedauer und Reiseweiten könnten sich erhöhen.

Neue Nutzergruppen: Beim automatisierten Fahren werden Fahraufgaben vom Fahrer an das System abgegeben, bei tieferen Automatisierungsstufen temporär und bei höheren Levels dauerhaft. In den höchsten Automatisierungsstufen kann das Steuern des Fahrzeuges durch den Menschen gänzlich entfallen. Damit werden die Anforderungen an den Fahrer gesenkt bzw. aufgehoben. Auch bisher mobilitätseingeschränkte Personen können Fahrzeuge ohne Führerschein und ohne Begleitung eines Fahrers nutzen. Dies betrifft insbesondere Kinder, Jugendliche und Senioren.

Leerfahrten: Sind Fahrzeuge vollautomatisiert unterwegs, muss kein Fahrer mehr an Bord sein. Vor allem bei der privaten Nutzung dürften so durch das Bringen und Abholen von Personen und Waren viele Leerfahrten entstehen. Aber auch bei automatisierten Sharing-Fahrzeugen müssten Fahrten ohne Pas- 
sagiere zwischen den Ab- und Zugangsorten durchgeführt werden. Entstehende Leerfahrten erhöhen die Fahrleistung und reduzieren den durchschnittlichen Besetzungsgrad.

Änderung der Verkehrsmittelwahl: Die Abschätzung der Auswirkungen auf den Modal Split ist am unsichersten, weil gegenläufige Effekte eintreten könnten. Im Zuge der Automatisierung werden sich die verkehrlichen Angebote in ihren Vor- und Nachteilen verändern. Die Verkehrsmittelwahl ist vor allem abhängig von den Preisen, den Reisezeiten und dem Komfort. Durch den Entfall des Fah-

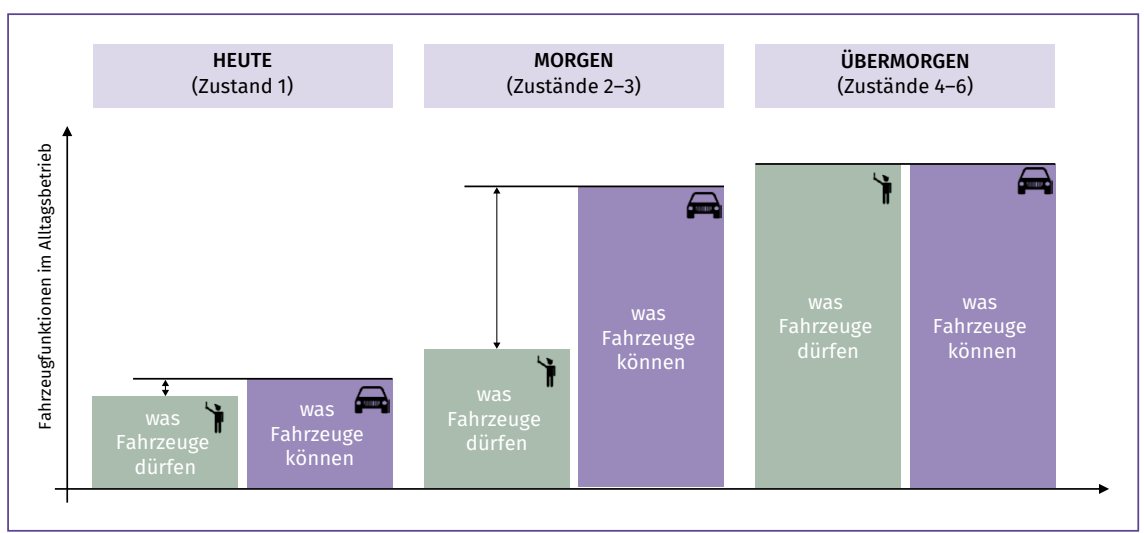

Abb.3: Diskrepanz zwischen den technologischen Möglichkeiten und der zugelassenen Nutzung.

Quelle: Eigene Darstellung bei privaten Sharing-Diensten deutliche

Kostenvorteile erreicht (Bösch et al. 2017). Infolge von tieferen Preisen gewinnen diese Angebote an Attraktivität. Beim MIV sind aber nicht die Gesamtkosten, sondern die variablen Kosten (z. B. Treibstoff) für die unmittelbare Verkehrsmittelwahl maßgebend. Und diese dürften weiterhin geringer ausfallen als die Preise der neuen Angebote (Bösch et al. 2017). Privatfahrzeuge weisen überdies einen höheren Komfort auf. Trotzdem dürfte vor allem der MIV in Bezug auf die Verkehrsleistung Anteile an die neuen automatisierten Angebote verlieren. Beim ÖV bestehen durch die Kosteneinsparungen im Zuge der Automatisierung (Entfall von Personal) verschiedene nachfragesteigernde Möglichkeiten: Bei gleich hohen Abgeltungsleistungen und Tarifen können mehr Leistungen bestellt und das Angebot somit ausgeweitet werden. Bei gleichbleibenden Abgeltungen und gleichem Angebot können Tarife gesenkt werden, was Umsteiger generiert.

Kapazitäten und Reisezeiten: Durch die Automatisierung kann die Kapazität der bestehenden Infrastruktur vergrößert werden, da geringere mittlere Folgezeitlücken zwischen automatisierten Fahrzeugen erreicht werden können. Simulationsresultate für Hochleistungsstraßen zeigen unter der Berücksichtigung von unterschiedlichen Streckenabschnitten, Durchdringungsraten automatisierter Fahrzeuge, Geschwindigkeitsregimes, Schwerverkehrsanteilen sowie Verkehrsstromverhältnissen Kapazitätswirkungen in der Größenordnung von 0 bis zu 30\% (Fellendorf 2017). Allerdings sind die Angaben zu Zeitlücken, die automatisierte Fahrzeuge einhalten können, noch nicht gesichert und von Annahmen zur Vernetzung der Fahrzeuge abhängig. Wenn automatisierte Fahrzeuge in den früheren Zuständen aufgrund von Sicherheitsüberlegungen höhere mittlere Zeitlücken einhalten, als dies heute der Fall ist, würde beispielsweise auf dem Autobahnnetz in Deutschland die Kapazität vorerst abnehmen (Hartmann et al. 2017). In Städten besteht bei vollständiger Durchdringung ein theoretisches Potenzial für Kapazitätssteigerungen von rund 20 bis $40 \%$ (Friedrich 2015). Dabei sind vor allem Knoten mit Lichtsignalanlagen maßgebend, an denen ein verringerter Zeitbedarf pro Fahrzeug, eine Harmonisierung der Zeitlücken, ein unverzögertes Anfahren sowie eine erhöhte Räumgeschwindigkeit infolge der Automatisierung erwartet werden. Aufgrund von zusätzlich zu berücksichtigenden Interaktionen unter den Verkehrsteilnehmenden dürfte die Kapazitätswirkung in der Stadt aber kleiner sein als auf dem Hochleistungsnetz. Die Kapazitätszunahmen können zu Mehrverkehr auf der Straße führen (neu generierte Fahrten, veränderte Zielwahl, Verkehrsträgerverlagerungen). Als Folge verändern sich Reisezeiten und Erreichbarkeiten im Verkehrssystem.

\section{Infrastruktur}

Automatisierte Fahrzeuge sind auf eine adäquate Datenübertragungsinfrastruktur angewiesen, damit die Potenziale für einen optimalen Betrieb der Verkehrsinfrastruktur genutzt werden können. Hierzu sollen Fahrzeuge untereinander und mit der Infrastruktur kommunizieren. Beispielsweise können so die Zeitlücken zwischen den Fahrzeugen weiter reduziert und die Kapazitäten erhöht werden (Hartmann et al. 2017). Zudem besteht die Möglichkeit, dass Ereignisdaten ausgetauscht werden, insbesondere zu Gefahren oder Stauzuständen auf dem Netz, oder dass das Verkehrsmanagement mit geeigneten Instrumenten erweitert wird, damit sich das Verkehrssystem einem Systemoptimum annähert. Voraussetzung, um diesen Nutzen zu realisieren, ist die Anpassung der digitalen Infrastruktur.

Bei den Straßenkapazitäten ist zwar eine Erhöhung infolge der Automatisierung möglich, diese steigen allerdings erst mit einer hohen Durchdringung automatisierter Fahrzeuge in relevantem Ausmaß an (Friedrich 2015). Damit zeichnen sich zumindest mittelfristig keine deutlichen Sprünge ab. Zudem ist bei der Leistungsfähigkeit immer der Abschnitt mit der geringsten Kapazität maßgebend, daher muss immer eine Netzbetrachtung erfolgen. Die Kapazitätseffekte können allenfalls punktuell helfen, den Bedarf an Infrastrukturausbauten zu reduzieren.

Im Siedlungsraum stehen Veränderungen bei der Verkehrsinfrastruktur an: Infolge der zunehmenden Nutzung von automatisierten Sharing-/Pooling-Fahrzeugen müssen genügend Haltemöglichkeiten definiert und rechtlich geregelt werden. Gleichzeitig könnte der Bedarf an Parkierungsflächen deutlich reduziert 
werden, wenn kollektive Angebote intensiv benutzt werden und auf das Privatfahrzeug verzichtet wird. Zu klären sind zusätzlich die Anforderungen an Straßenmarkierungen und Verkehrssignale.

Das Nebeneinander verschiedener Verkehrsmittel nimmt insbesondere im Siedlungsgebiet zu: Es wird nicht mehr nur zwischen Fußverkehr, Radverkehr, ÖPNV und MIV mit ihren Ansprüchen an den Verkehrsraum zu unterscheiden sein. Einerseits werden neue Mischformen zwischen ÖPNV und MIV auftreten, die weitere Ansprüche an den öffentlichen Raum mitbringen. Andererseits ist beim motorisierten Verkehr auch zwischen den verschiedenen Automatisierungsgraden, deren Anforderungen sowie deren Vorschriften und Rahmenbedingungen zu unterscheiden. Es ist davon auszugehen, dass Fahrzeuge während langer Zeiträume auf unterschiedlichen Automatisierungsstufen im gleichen Raum unterwegs sind. Diese Mischverkehre dürften unseren Alltag in den kommenden Jahrzehnten prägen und sich auf das Erscheinungsbild der Mobilität in Siedlungsräumen auswirken. Die innerstädtischen Straßenräume müssen an die Anforderungen dieses Mischverkehrs zwischen automatisierten und nicht automatisierten Fahrzeugen sowie weiteren Verkehrsteilnehmenden, insbesondere dem Fuß- und Radverkehr, angepasst werden.

\section{Kollektiver Personenverkehr}

Der straßengebundene ÖPNV erhält durch die Vollautomatisierung neue Perspektiven. Längerfristig besteht die Möglichkeit, die Mobilität nachfrageorientierter zu gestalten. In nachfrageschwachen Gebieten können Linien aufgehoben und das Routing adaptiv auf die Kundenwünsche gestaltet werden. Durch selbstfahrende Fahrzeuge ergeben sich im Bereich des Sharings und Poolings neue Möglichkeiten zur Organisation der Transportketten. Automatisierte Fahrzeuge können durch entsprechende Weg-Anmeldungen kollektiv oder individuell verwendet werden. Mit der Automatisierung verschärft sich der Wettbewerb zwischen den verschiedenen Verkehrsmitteln. Eine Veränderung des Modal Splits ist die wahrscheinliche Folge.

Gefragt sind auch intermodale Plattformen, welche die öffentlichen und privaten Transportleistungen kombinieren sowie Wegeketten mit verschiedenen Verkehrsmitteln organisieren und die Bezahlung über ein einziges Konto regeln. Für öffentliche Transportunternehmen stellt sich damit bereits heute die Frage, inwiefern sie die Chancen solcher Mobilitätsdienste (Mobilityas-a-Service) nutzen können.

\section{Fazit und Handlungsbedarf}

\section{Chancen und Herausforderungen}

Aus Sicht der Städte und Regionen bietet das automatisierte Fahren zahlreiche Chancen: Pooling- und Sharing-Fahrzeuge führen zu Effizienz- und Flächengewinnen beim Parkraum. Bei einer Reduktion von Folgezeitlücken sind Kapazitätsgewinne und eine erhöhte Stabilität des Verkehrsflusses auf der Straße möglich. Bei hoher Durchdringung mit automatisierten Fahrzeugen können allenfalls Knotensteuerungen angepasst werden. Durch das Vermeiden menschlicher Fehler könnte die Verkehrssicherheit erhöht werden. Automatisierte Fahrzeuge ermöglichen eine verbesserte, auf einer Datenkommunikation basierte Verkehrssteuerung. Mobility-as-a-Service-Angebote können die individuelle Mobilität erleichtern. Neue Angebote im ÖPNV und Sharing-Fahrzeuge verbessern die Erschließungsqualität und ermöglichen Mobilität für bisher mobilitätseingeschränkte Nutzergruppen.

Die Herausforderungen bestehen vor allem in der potenziellen Zunahme der verkehrlichen Nachfrage und der damit verbundenen Erhöhung der Fahrleistung. Verschiedene treibende Nachfrageeffekte überlagern sich: Mit der (Voll-)Automatisierung sind Leerfahrten möglich, es entstehen neue Transportangebote, und ohne die Notwendigkeit eines Fahrers werden auch Nutzer ohne klassischem Führerschein in automatisierten Fahrzeugen unterwegs sein können. Kapazitätserhöhungen infolge der Automatisierung führen zu Mehrverkehr. Zudem könnten diese die Erreichbarkeiten so verändern, dass insbesondere ländliche Gemeinden und Agglomerationen profitieren, während Städte an Attraktivität einbüßen (Meyer et al. 2016). Folge davon ist eine Zunahme der Zersiedelung. Was also müssen die Behörden auf allen Stufen tun und wie müssen sie sich abstimmen, um Chancen zu nutzen und Herausforderungen anzugehen (Abb.4)? Wie können sich ÖPNV-Anbieter und Autohersteller positionieren?

\section{Mobilitäts- und Verkehrsmanagement}

Behörden können mit verschiedenen verkehrsplanerischen, -technischen und -politischen Maßnahmen lenkend eingreifen und mögliche Fehlentwicklungen korrigieren. Insbesondere müssen Instrumente gefunden werden, welche die potenzielle Erhöhung der Fahrleistungen infolge der nutzbaren Reisezeiten abschwächen sowie das Ausmaß von Leerfahrten automatisierter Fahrzeuge beeinflussen. Leerfahrten von neuen Sharing- und Pooling-Angeboten dürften kaum zu verhindern sein, sie haben jedoch - insbesondere im MIV - das Potenzial, weitere Verkehrszusammenbrüche zu provozieren. Dabei sind Zufahrtsbeschränkungen für leere Fahrzeuge, ein Mobility Pricing oder Subventionen für Pooling denkbar. Weiter könnte zum Beispiel in Zonen, wo ein hohes Verkehrsaufkommen regelmäßig zu Staus führt, der Einsatz von vollautomatisierten Fahrzeugsystemen für entsprechend ausgerüstete Fahrzeuge zur Pflicht werden. Auch muss die kollektive Fahrzeugnutzung mit geeigneten Anreizen

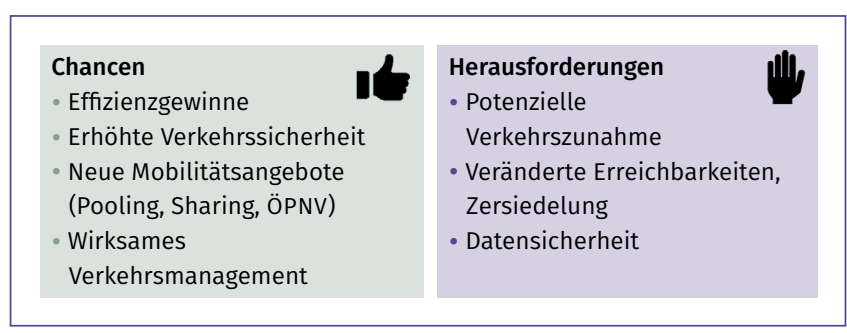

Abb.4: Chancen und Herausforderungen des automatisierten Fahrens. Quelle: Eigene Darstellung 
gefördert werden. Die staatlichen Abgeltungen im ÖV müssen auf die neue Konkurrenzsituation von Verkehrsmitteln ausgerichtet werden. In welchem Ausmaß solche Maßnahmen im Mobilitätsund Verkehrsmanagement von der Gesellschaft akzeptiert und umgesetzt werden können, wird letztlich von der jeweiligen nationalen, regionalen und kommunalen Verkehrspolitik abhängig sein und sich erst noch zeigen müssen.

\section{Handlungsbedarf}

Die Auseinandersetzung mit dem Entwicklungspfad führt zu ganz unterschiedlichen Erkenntnissen. Bereits die der Studie zugrunde gelegten Annahmen zeigen, wie vielfältig die Zukunft des automatisierten Fahrens tatsächlich aussehen könnte. Letztlich müssen wir als Gesellschaft diskutieren, welche Vorteile des automatisierten Fahrens wir nutzen und welche unerwünschten Entwicklungen wir vermeiden wollen. Die Vorteile von automatisierten Fahrzeugen wie Sicherheits-, Effizienz-, Zeit- und Komfortgewinne stellen sich nicht von selbst ein.

Voraussetzungen sind erstens eine geeignete Praxis bei der Zulassung von Fahrzeugen und Software, bei Haftungsfragen sowie bei der Ausstellung von Führerscheinen, Nutzer- oder Betreiberausweisen. Zweitens sind attraktive sowie akzeptierte Sharing- und Pooling-Angebote zur Individualisierung und Nachfrageadaption im kollektiven Verkehr notwendig. Die zunehmenden Mobilitätsansprüche können vor allem dann nachhaltig abgewickelt werden, wenn die Auslastung der Fahrzeuge erhöht wird. Drittens sind griffige Instrumente im Verkehrsmanagement zu finden, welche die verfügbaren Straßenkapazitäten optimal ausnutzen. Viertens muss die Vernetzung vorangetrieben werden, indem eine entsprechende Dateninfrastruktur aufgebaut wird.

Werden diese Voraussetzungen nicht rechtzeitig geschaffen, so resultiert eine klare Zunahme der Fahrleistungen insbesondere infolge von Leerfahrten, von Komfortsteigerung und Zeitgewinnen. Die Rahmenbedingungen müssen schrittweise und wirkungsvoll gesetzt werden. Dem politischen Gestaltungswillen und dem gesellschaftlichen Diskurs kommt also ein zentraler Stellenwert zu. Ohne angemessene Regelungen von Zustand zu Zustand sind Fehlentwicklungen wie zunehmende Staus und Verkehrsbehinderungen absehbar.

\section{Literatur}

Bösch, Patrick; Becker, Felix; Becker, Henrik; Axhausen, Kay (2017): Cost-based analysis of autonomous mobility services. Working Paper. ETH Zurich: Institute for Transport Planning and Systems (IVT).

Deutsche Bundesregierung (2015): Strategie automatisiertes und vernetztes Fahren. Leitanbieter bleiben, Leitmarkt werden, Regelbetrieb einleiten. Berlin: Bundesministerium für Verkehr und digitale Infrastruktur.

EBP - Ernst Basler + Partner (2017): Einsatz automatisierter Fahrzeuge im Alltag. Denkbare Anwendungen und Effekte in der Schweiz. Schlussbericht Grundlagenanalyse (Phase A). Zürich: EBP Schweiz AG.

Fellendorf, Martin (2017): Automatisierung im Mischverkehr. Verkehrswissenschaftliche Erkenntnisse, Überlegungen zur Leistungsfähigkeit. Technische Universität Graz: ÖVG-Forum Automatisierung im Verkehr.
Friedrich, Bernhard (2015): Verkehrliche Wirkung autonomer Fahrzeuge. In: Autonomes Fahren. Technische, rechtliche und gesellschaftliche Aspekte. Wiesbaden: Springer, S. 331-350, DOI: 10.1007/978-3-662-45854-9.

Hartmann, Martin; Motamedidehkordi, Nassim; Krause, Sabine; Hoffmann, Silja; Vortisch, Peter; Busch, Fritz (2017): Impact of automated vehicles on capacity of the German freeway network. Montreal: Conference Paper, ITS World Congress.

Meyer, Jonas; Becker, Henrik; Bösch, Patrick; Axhausen, Kay (2016): Impact of autonomous vehicles on the accessibility in Switzerland. ETH Zürich: Working Paper, Institute for Transport Planning and Systems (IVT).

OECD/ITF - Organisation für wirtschaftliche Zusammenarbeit und Entwicklung/ International Transport Forum (2015): Urban mobility system upgrade. How shared self-driving cars could change city traffic. Paris: International Transport Forum.

SAE International (2014): Automated driving, levels of driving automation are defined in New SAE International Standard J3016. Warrendale: Society of Automotive Engineers.

Schweizerischer Bundesrat (2016): Automatisiertes Fahren. Folgen und verkehrspolitische Auswirkungen. Bericht des Bundesrates in Erfüllung des Postulats Leutenegger Oberholzer 14.4169 „Auto-Mobilität", 0353-1246. Bern: Schweizer Eidgenossenschaft.

UITP - Internationaler Verband für öffentliches Verkehrswesen (2017): Policy brief, autonomous vehicles. A potential game changer for urban mobility. Brüssel: International Association of Public Transport.

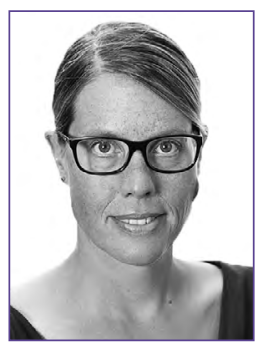

\section{FABIENNE PERRET}

ist Leiterin des Geschäftsbereichs Verkehr bei EBP Schweiz. Sie hat langjährige Erfahrung in der Planung und Beratung zu Gesamtverkehrs- und Mobilitätsfragen, sowohl für die öffentliche Hand als auch für private Unternehmen. Sie studierte Geomatikingenieurwissenschaften in Zürich und Victoria.

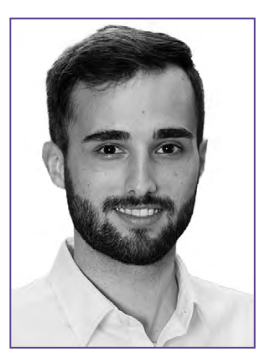

\section{REMO FISCHER}

arbeitet im Geschäftsbereich Verkehr bei EBP Schweiz mit den Schwerpunkten Verkehrsplanung und Verkehrstechnik. Als Bauingenieur erforscht er das Verhalten von Verkehrssystemen und wendet sein Fachwissen in der Planung und Projektierung an

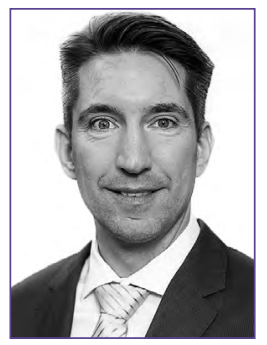

\section{HOLGER FRANTZ}

ist Geschäftsführer von EBP Deutschland. Er beschäftigt sich insbesondere mit Auswirkungsanalysen des Verkehrs, der Analyse von lufthygienischen und stadtklimatischen Situationen, mit Elektromobilität und weiteren zukünftigen Mobilitätsformen. 